\title{
Correspondence
}

\section{Is PDPH from a 25-gauge Whitacre needle always short-lasting and self- resolving?}

To the Editor:

With the development of small gauge pencil-point needles, the incidence of postdural puncture headache (PDPH) in obstetric patients has diminished considerably. Most of the studies on PDPH focus on its incidence and prevention. ${ }^{1}$ In our hospital, the majority of Cesarean deliveries are performed under spinal anesthesia for which we use only 25-gauge Whitacre spinal needles (Becton-Dickinson, Madrid, Spain). Recently, two patients developed PDPH after spinal anesthesia.

A 35-yr-old parturient received spinal anesthesia with a 25 -gauge Whitacre spinal needle at L3-4 interspace. At the $24 \mathrm{hr}$ follow-up, the patient had no complaints. At $48 \mathrm{hr}$ after the spinal injection, she developed severe postural headache not relieved with propoxyphene and paracetamol and associated with neck pain. The headache improved in the evening, became mild on the next day and resolved completely on the third day.

Another 35-yr-old parturient underwent an uneventful spinal anesthesia with a 25 -gauge Whitacre spinal needle at the L3-4 interspace. Similar to the first patient, she developed severe postural headache not relieved with propoxyphene and paracetamol 48 $\mathrm{hr}$ after the spinal injection. Her headache became mild four hours afterwards and disappeared completely on the following day.

There has been extensive research on the pathogenesis, prevention and treatment of PDPH. ${ }^{2}$ There is, however, a paucity of information on the clinical course of PDPH secondary to the use of different needles. In the meta-analysis of obstetrical studies on PDPH by Choi's group, PDPH had an onset of one to seven days after dural puncture and lasted from 12 hr to seven days, but PDPH was not characterized according to type of needle. ${ }^{3}$ In a study comparing five spinal needles, the use of 25 -gauge Whitacre needles was associated with a $3.1 \%$ incidence of PDPH and $0 \%$ blood patch. ${ }^{4}$ Epidural blood patch for PDPH is not without risks and timing of the procedure remains controversial and non-standardized. Studies on the clinical course of PDPH including the onset, severity and duration are desirable. It would be reas- suring to know if PDPH from a small gauge pencilpoint spinal needle such as the Whitacre needle is mostly self-resolving within a short period of time, obviating the need for an epidural blood patch.

Andrew Y.C. Wong FHKAM

Hong Kong, China

\section{References}

1 Choi PT, Galinski SA, Lucas S, Takeuchi L, Jadad AR. Examining the evidence in anestheisa literature: a survey and evaluation of obstetrical postdural puncture headache reports. Can J Anesth 2002; 49: 49-56.

2 Turnbull DK, Shepherd DB. Post-dural puncture headache: pathogenesis, prevention and treatment. Br J Anaesth 2003; 91: 718-29.

3 Choi PT, Galinski SE, Takeuchi L, Lucas S, Tamayo C, Jadad AR. PDPH is a common complication of neuraxial blockade in parturients: a meta-analysis of obstetrical studies. Can J Anesth 2003; 50: 460-9.

4 Vallejo MC, Mandell GL, Sabo DP, Ramanathan S. Postdural puncture headache: a randomized comparison of five spinal needles in obstetric patients. Anesth Analg 2000; 91: 916-20.

\section{REPLY:}

I agree with $D r$. Wong's observations on the lack of information relating to the details regarding postdural puncture beadache (PDPH) in clinical reports despite the extensive amount of literature on this topic. At the time of the meta-analysis, 12 reports had been published on PDPH with Whitacre 25-gange needles in the obstetrical population. Of the 6,366 patients studied, 103 suffered PDPHs yielding a pooled estimate of 2.2\% (adjusted for within-study and between-study variances). ${ }^{1}$ Of the 103 PDPHs, the headache was described as mild, moderate, and severe in 16, 21, and seven patients respectively. ${ }^{2}$ The intensity of the remaining 59 headaches was not reported. Seven studies reported the duration of followup (median 5 days, range 1 to 42 days). ${ }^{2}$ Onset of $P D P H$, reported in only one study, ranged from one to five days post-dural puncture with 8 mild, 11 moderate, and 6 severe PDPHs. ${ }^{2}$ Duration, also reported in only one study, was one day in one PDPH of unknown intensity. ${ }^{2}$ Neither study reported duration of follow-up. Two studies described the interventions used to treat PDPH. ${ }^{2}$

Over 100 years of observational and experimental data have been published on PDPH. The International 